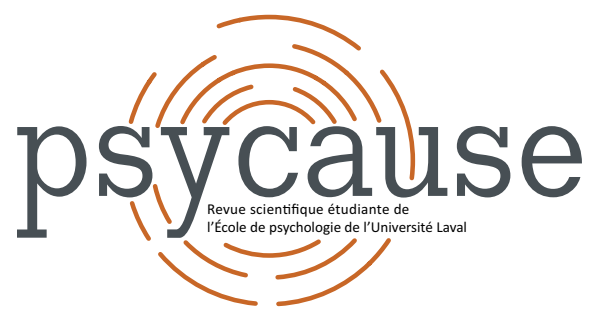

\title{
PSYCAUSE
}

Revue scientifique étudiante de l'École de psychologie de l'Université Laval

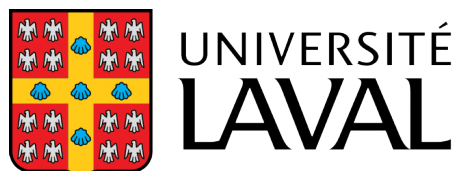

Faculté des sciences sociales École de psychologie

\section{LA RELATION ENTRE LES PENSÉES POSITIVES ET NÉGATIVES, LES STRATÉGIES DE RÉGULATION ÉMOTIONNELLE ET LA PEUR DE RÉCIDIVE DU CANCER}

\author{
Samuele DALLAIRE-HABEL ${ }^{1, *}$, Geneviève OUELLET ${ }^{1}$, Frédéric THÉRIAULT-COUTURE¹, \\ Véronique MASSICOTTE ${ }^{1,2,3}$, Hans IVERS ${ }^{1,2,3}$, Anne-Josée GUIMOND ${ }^{1,2,3}$ et Josée SAVARD ${ }^{1,2,3}$ \\ 'École de Psychologie, Université Laval \\ ${ }^{2}$ Centre de recherche du CHU de Québec-Université Laval \\ ${ }^{3}$ Centre de recherche sur le cancer de l'Université Laval \\ * samuele.dallaire-habel.1@ulaval.ca
}

\section{Pour citer l'article}

Dallaire-Habel, S., Ouellet, G., Thériault-Couture, F., Massicotte, V., Ivers, H., Guimond, A.-J., \& Savard, J. (2019). La relation entre les pensées positives et négatives, les stratégies de régulation émotionnelle et la peur de récidive du cancer. Psycause: Revue scientifique étudiante de l'École de psychologie de l'Université Laval, 9(2), 15-17. 
Millar, W. S., \& Weir, C. (2015). Baseline response levels are a nuisance in infant contingency learning. Infant and Child Development, 24(5), 506-521.

Neuhauser, A. (2018). Predictors of maternal sensitivity in at-risk families. Early Child Development and Care, 188(2), 126142.

Northrup, J. B. (2017). Contingency detection in a complex world: A developmental model and implications for atypical development. International Journal of Behavioral Deve- lopment, 41(6), 723-734.

Spieker, S. J., Larson, N. C., Lewis, S. M., White, R. D., \& Gilchrist, L. (1997). Children of adolescent mothers: Cognitive and behavioral status at age six. Child and Adolescent Social Work Journal, 14(5), 335-364.

Sullivan, M. W., \& Lewis, M. (2003). Contextual determinants of anger and other negative expressions in young infants. Developmental psychology, 39(4), 693.

\title{
Pour citer l'article
}

Fortin, L., St-Pierre, C., St-Pierre, É., Langlois, É., \& Tarabulsy, G. M. (2019). La détection des contingences chez les enfants âgés entre six et 18 mois. Psycause: Revue scientifique étudiante de l'École de psychologie de l'Université Laval, 9(2), 13-15.

\section{LA RELATION ENTRE LES PENSÉES POSITIVES ET NÉGATIVES, LES STRATÉGIES DE RÉGULATION ÉMOTIONNELLE ET LA PEUR DE RÉCIDIVE DU CANCER}

\author{
Samuele DALLAIRE-HABEL ${ }^{1, *}$, Geneviève OUELLET' ${ }^{1}$, Frédéric THÉRIAULT-COUTURE ${ }^{1}$, Véronique MASSICOTTE ${ }^{1,2,3}$, \\ Hans IVERS ${ }^{1,2,3}$, Anne-Josée GUIMOND ${ }^{1,2,3}$ et Josée SAVARD ${ }^{1,2,3}$ \\ ${ }^{1}$ École de Psychologie, Université Laval; ${ }^{2}$ Centre de recherche du CHU de Québec-Université Laval; ${ }^{3}$ Centre de recherche sur \\ le cancer de l'Université Laval \\ *samuele.dallaire-habel.1@ulaval.ca
}

\section{Mots-clés : Cancer; Peur de la récidive du cancer; Pensées; Régulation émotionnelle; Modèle cognitif}

La peur de la récidive du cancer (PRC), qui se définit comme la peur, l'inquiétude ou une préoccupation quant à la possibilité que le cancer revienne ou progresse, est fréquemment rapportée par les patientes atteintes d'un cancer du sein (Lebel et coll., 2016). Certains travaux ont examiné le rôle des pensées dans la PRC et montrent qu'une plus grande tendance à entretenir des pensées négatives et à anticiper négativement le futur (pessimisme) est positivement associée à la PRC (p. ex., Deimling, Bowman, Sterns, Wagner \& Kahana, 2006). II a été proposé dans la littérature dédiée au grand public que la pensée positive pourrait s'avérer une option de rechange avantageuse. Toutefois, une étude sur l'optimisme irréaliste, un construit apparenté, suggère qu'elle pourrait être associée à de la détresse psychologique (Hurt, Weinman, Lee \& Brown, 2012). Par ailleurs, le modèle des processus de régulation émotionnelle (RE) de Gross et Thompson (2007) souligne la pertinence d'examiner le rôle médiateur des stratégies de RE dans la relation entre les pensées et la PRC en proposant que l'interprétation cognitive d'une situation amène l'individu à adopter certaines stratégies de RE afin d'exercer une influence sur ses émotions. La présente étude, menée chez des femmes atteintes d'un cancer du sein, a pour objectifs: (a) d'examiner les associations transversales et prospectives entre les pensées négatives et positives et la PRC; et (b) d'explorer le rôle médiateur des stratégies de $R E$, soit la réévaluation cognitive, la suppression émotionnelle et l'évitement expérientiel, dans la relation entre le type de pensées (négatives et positives) et la PRC.

\section{Méthode}

L'échantillon est composé de 81 femmes recrutées à l'HôtelDieu de Québec (Centre hospitalier universitaire de QuébecUniversité Laval), ayant reçu un diagnostic de cancer du sein. Les participantes sont âgées en moyenne de 59 ans (É-T $=8,5$ ) et la majorité est traitée pour un cancer de stade 1 $(66,3 \%)$. Les patientes complètent l'Inventaire de peur de récidive du cancer, le Questionnaire des pensées et anticipations au sujet du cancer (PAC), le Questionnaire de régulation émotionnelle et le Questionnaire d'acceptation et d'action-Il à la maison avant le début des traitements de radiothérapie (T1) et après leur fin ( $T 2$; six semaines après le T1). Puisque le PAC mesure les pensées et anticipations quant au traitement à recevoir, il est administré au T1 uniquement. 
d'occurrence des pensées négatives explique une portion de la relation positive entre le recours à l'évitement expérientiel ainsi qu'à la suppression émotionnelle, et la PRC. Les résultats suggèrent aussi que la fréquence d'occurrence des pensées positives explique une partie de la relation positive entre le recours à la suppression émotionnelle et la PRC. Ces observations sont cohérentes avec celles de David, Montgomery et Bovbjerg (2006) qui montrent que le pessimisme et l'optimisme dispositionnel médient la relation entre les stratégies de RE et la détresse psychologique chez ces patientes atteintes. Ainsi, il semble que les stratégies de RE utilisées par les participantes pour agir sur leur PRC les amèneraient à modifier le type et la fréquence d'occurrence de leurs pensées.

En somme, ces résultats remettent en doute les effets bénéfiques de la pensée positive dans l'adaptation psychologique au cancer et identifient un mécanisme possible par lequel les stratégies de RE et les pensées accroîtraient le niveau de sévérité de la PRC chez les femmes atteintes d'un cancer du sein. Ils appuient la pertinence de s'intéresser à l'efficacité des interventions cognitives-comportementales pour diminuer la PRC en soulignant l'importance des interprétations cognitives. D'autres études seront nécessaires afin de mieux comprendre les relations entre ces variables.

\section{Références}

David, D., Montgomery, G. H., \& Bovbjerg, D. H. (2006). Relations between coping responses and optimism-pessimism in predicting anticipatory psychological distress in surgical breast cancer patients. Personality and Individual Differences, 40(2), 203-213. doi:10.1016/j.paid.2005.05.018

Deimling, G. T., Bowman, K. F., Sterns, S., Wagner, L. J., \& Kahana, B. (2006). Cancer-related health worries and psychological distress among older adult, long-term cancer survivors. Psycho-Oncology, 15(4), 306-320. doi:10.1002/ pon.955

Gross, J. J., \& Thompson, R. A. (2007). Emotion Regulation: Conceptual Foundations. New York, NY: Guilford Press.

Hurt, C. S., Weinman, J., Lee, R., \& Brown, R. G. (2012). The relationship of depression and disease stage to patient perceptions of Parkinson's disease. Journal of Health Psychology, 17(7), 1076-1088. doi: 10.1177/1359105311428537

Lebel, S., Ozakinci, G., Humphris, G., Mutsaers, B., Thewes, B., Prins, J., Dinkel, A., \& Butow, P. (2016). From normal response to clinical problem: definition and clinical features of fear of cancer recurrence. Supportive Care in Cancer, 24(8), 3265-3268. doi:10.1007/s00520-016-3272-5

Savard, J. (2010). Faire face au cancer avec la pensée réaliste. Montréal, QC: Flammarion.

\title{
Pour citer l'article
}

Dallaire-Habel, S., Ouellet, G., Thériault-Couture, F., Massicotte, V., Ivers, H., Guimond, A.-J., \& Savard, J. (2019). La relation entre les pensées positives et négatives, les stratégies de régulation émotionnelle et la peur de récidive du cancer. Psycause: Revue scientifique étudiante de l'École de psychologie de l'Université Laval, 9(2), 15-17.

\section{LANGUE D'ORIGINE ET EXPÉRIENCE IDENTITAIRE CHEZ LES CANADIENS DE DEUXIÈME GÉNÉRATION}

\author{
Milica MILJUS ${ }^{1}$ $*$, Frédérique MONTREUIL ${ }^{1}$, Laurence GARCEAU ${ }^{1}$, Jennifer TAING ${ }^{1}$ et Maya A. YAMPOLSKY ${ }^{1}$ \\ ${ }^{1}$ École de psychologie, Université Laval \\ *milica.miljus.1@ulaval.ca
}

\section{Mots clés : Langue d'origine; Deuxième génération; Identité; Sentiment d'appartenance}

Les individus de deuxième génération sont définis comme étant nés au pays et ayant au moins un parent migrant (Nesteruk, Helmstetter, Gramescu, Siyam \& Price, 2015). Les enfants qui ont immigré avant l'âge de cinq ans sont inclus dans cette définition, ayant été socialisés dans la culture majoritaire de façon similaire à ceux nés au pays (Nesteruk et coll., 2015). Les individus de deuxième génération tendent à adopter la langue majoritaire par le biais de l'environnement social (p. ex., à l'école), et ce, au détriment du maintien ou de l'apprentissage de la langue d'origine (Fillmore, 1991). Sachant que la connaissance de la langue d'origine joue un rôle dans le développement et le renforcement de l'identité culturelle (Phinney, Romero, Nava \& Huang, 2001), cette recherche qualitative vise à explorer les relations entre l'apprentissage de la langue, le sentiment d'appartenance et l'expérience identitaire des Canadiens de deuxième génération et à explorer les variables contextuelles associées. 